\title{
Antifungal Combinations in Dermatophytes
}

\author{
Lucia Brescini ${ }^{1}$, Simona Fioriti ${ }^{1}$, Gianluca Morroni ${ }^{1}$ (1) and Francesco Barchiesi ${ }^{1,2, *}$ \\ 1 Dipartimento di Scienze Biomediche e Sanità Pubblica, Università Politecnica delle Marche, \\ 60020 Ancona, Italy; 1.brescini@univpm.it (L.B.); s.fioriti@staff.univpm.it (S.F.); g.morroni@univpm.it (G.M.) \\ 2 Malattie Infettive, Azienda Ospedaliera Ospedali Riuniti Marche Nord, 61121 Pesaro, Italy \\ * Correspondence: f.barchiesi@univpm.it; Tel.: +39-721-36-5505
}

Citation: Brescini, L.; Fioriti, S.; Morroni, G.; Barchiesi, F. Antifungal Combinations in Dermatophytes. J. Fungi 2021, 7, 727. https://doi.org/ $10.3390 /$ jof7090727

Academic Editors: Patrick Schwarz and Eric Dannaoui

Received: 10 August 2021

Accepted: 1 September 2021

Published: 5 September 2021

Publisher's Note: MDPI stays neutral with regard to jurisdictional claims in published maps and institutional affiliations.

Copyright: () 2021 by the authors. Licensee MDPI, Basel, Switzerland. This article is an open access article distributed under the terms and conditions of the Creative Commons Attribution (CC BY) license (https:// creativecommons.org/licenses/by/ $4.0 /)$.

\begin{abstract}
Dermatophytes are the most common cause of fungal infections worldwide, affecting millions of people annually. The emergence of resistance among dermatophytes along with the availability of antifungal susceptibility procedures suitable for testing antifungal agents against this group of fungi make the combinatorial approach particularly interesting to be investigated. Therefore, we reviewed the scientific literature concerning the antifungal combinations against dermatophytes. A literature search on the subject performed in PubMed yielded 68 publications: 37 articles referring to in vitro studies and 31 articles referring to case reports or clinical studies. In vitro studies involved over 400 clinical isolates of dermatophytes (69\% Trichophyton spp., 29\% Microsporum spp., and 2\% Epidermophyton floccosum). Combinations included two antifungal agents or an antifungal agent plus another chemical compound including plant extracts or essential oils, calcineurin inhibitors, peptides, disinfectant agents, and others. In general, drug combinations yielded variable results spanning from synergism to indifference. Antagonism was rarely seen. In over 700 patients with documented dermatophyte infections, an antifungal combination approach could be evaluated. The most frequent combination included a systemic antifungal agent administered orally (i.e., terbinafine, griseofulvin, or azole-mainly itraconazole) plus a topical medication (i.e., azole, terbinafine, ciclopirox, amorolfine) for several weeks. Clinical results indicate that association of antifungal agents is effective, and it might be useful to accelerate the clinical and microbiological healing of a superficial infection. Antifungal combinations in dermatophytes have gained considerable scientific interest over the years and, in consideration of the interesting results available so far, it is desirable to continue the research in this field.
\end{abstract}

Keywords: dermatophytes; antifungals; antifungal susceptibility testing; drug combinations

\section{Introduction}

Dermatophytes are the most common cause of fungal infections worldwide, affecting millions of people annually. Dermatophytes are filamentous fungi with the ability to invade keratinised tissue, such as skin, hair, and nails [1]. Classically, they are divided into three genera: Trichophyton, Epidermophyton, and Microsporum [2]. However, this classification is based on the phenotype of the species and led to misclassification of morphological mutants. In 2017, de Hoog et al. constructed a phylogenetic tree using sequences of the nuclear ribosomal internal transcribed spacers (ITS rDNA) and divided the dermatophytes into seven clades: Trichophyton, Epidermophyton, Nannizzia, Paraphyton, Lophophyton, Microsporum, and Arthroderma [3]. Based on their host specificity, these fungi are classified into three ecological groups: geophilic, zoophilic, and anthropophilic. Geophilic dermatophytes rarely cause infection in animals and humans but may be carried by animals in their fur. Zoophilic dermatophytes occur in the fur of animal hosts, either symptomatically or asymptomatically, and can be easily transmitted to humans. When zoophilic and geophilic species are transmitted to humans, they cause acute, inflammatory mycoses. Transmission of anthropophilic dermatophytes is usually from human to human. They cause chronic, 
mild, noninflammatory infections [4,5]. Ringworm or tinea is one of the most frequent clinical aspect of dermatophytosis. Among the tinea infections, tinea corporis, tinea cruris, tinea pedis, and onychomycosis are the most predominant types. The dermatophytes T. rubrum, T. interdigitale and T. mentagrophytes, are the main aetiological agents of dermatophytosis of skin and nails in humans [1-5].

Medical treatment of dermatophytosis consists of topical or oral antifungal agents. There are many topical agents for treating several less severe forms of tinea [6]. The azole derivatives, such as clotrimazole, miconazole, econazole, and oxiconazole, are the generally used. Agents from the allylamine family, such as terbinafine and naftifine, are also used. Other topical agents, such as ciclopirox or amorolfine, can be effective in the less severe cases of onychomycosis. In the more severe forms of dermatophyte infections, oral treatment is generally employed [6]. The first oral agent used to treat a dermatophyte infection was griseofulvin, introduced in clinical practice in 1958 [7]. This molecule interferes with microtubule formation, thus impairing fungal growth and cell division. Allylamines (mainly terbinafine) and triazoles (mainly itraconazole) are used for oral therapy. Both allylamines and triazoles act on the same cellular target, that is, the cell membrane. Triazoles inhibit sterol 14- $\alpha$-demethylase, and allylamines inhibit squalene epoxidase, the inhibition of both enzymes leading to inhibition of ergosterol biosynthesis. Allylamines also lead to the accumulation of lanosterol, a toxic intermediary compound of the ergosterol biosynthesis pathway [8-10]. Terbinafine, which acts as a fungicide, is the drug of choice against Trichophyton spp. because of its clinical efficacy [11]. However, in the last years, an increasing incidence of chronic and recalcitrant dermatophytic infections have been described. Although rare, resistance to terbinafine has been documented among isolates of T. rubrum and T. mentagrophytes/T. interdigitale complex [12]. The resistance is generally due to several point mutations in the squalene target gene. This phenomenon, first described in recalcitrant dermatophytosis observed in India, was later reported in other countries [12-17]. Due to a very limited number of antifungals effective against dermatophytes and the emergence of resistance to these drugs, an in vitro antifungal susceptibility testing should be implemented in reference laboratories to monitor this phenomenon.

Currently, two standardized techniques for in vitro antifungal susceptibility testing of dermatophytes based on a broth microdilution procedure are available: one from the Clinical Laboratory Standards Institute (CLSI) and the other from the European Committee on Antimicrobial Susceptibility Testing (EUCAST) [18,19]. Although very similar, the two methods differ in endpoint determination. Lately, the EUCAST method was validated in a multicentre study (10 laboratories) in which terbinafine, itraconazole, voriconazole, and amorolfine were tested against a blinded panel of 38 terbinafine wild types and target gene mutant isolates of T. rubrum and T. interdigitale. The higher interlaboratory reproducibility was obtained using a medium with the addition of chloramphenicol and cycloheximide and measuring the MIC spectrophotometrically at 50\% inhibition [20].

An antifungal combination strategy has been lately implemented to overcome the resistance phenomenon against a wide variety of infections due to either yeasts or filamentous fungi [21,22]. Achievement of a synergistic interaction is desirable in these contexts. The emergence of resistance among dermatophytes along with the availability of antifungal susceptibility procedures suitable for testing antifungal agents against this group of fungi make the combinatorial approach particularly interesting to be investigated. Therefore, we aimed to review the scientific literature concerning the antifungal combinations used against dermatophytes. In order to include most of the published papers on this topic, the revision was carried out using the classic dermatophyte nomenclature, which divides these fungi into three genera. In particular, the results of in vitro combinations of several antifungals or antifungals plus other chemical compounds are presented. Additionally, the effects of combinatorial regimens in human infections are reported. 


\section{Materials and Methods}

This systematic review was conducted in accordance with the PRISMA guidelines [23] (Figure 1). PubMed was searched for dermatophytes antifungal combinations therapy with the following search string: "trichophyton" and "antifungal" and "combination"; "microsporum" and "antifungal" and "combination"; "epidermophyton" and "antifungal" and "combination". Literature search was conducted on 1 June 2021, by three individual researchers (L.B., S.F., and G.M.). In case of discrepancies in the process of inclusion of papers/data extraction, a consensus was reached through discussion or involvement of a fourth reviewer (F.B.). Additional cases were sought from the reference list of included papers. The inclusion criteria were antifungal combinations for Trichophyton spp., Microsporum spp., and Epidermophyton floccosum. The exclusion criteria were papers not referring to human studies (i.e., veterinary cases), papers in languages other than English, unreachable publications, papers not specifying the genera/species of dermatophytes, reviews of the literature, combinations considering two chemical compounds other than antifungals, and combinations not considering chemical compounds (i.e., photodynamic therapy). Data from the included papers were entered in a database, created with Excel, which encompassed the genus/species/number of dermatophytes tested, the type of drug combination, the method utilized for testing, and the results of the interaction. In case of clinical reports, demographic data (when available) and outcome of the combination therapy were also reported.

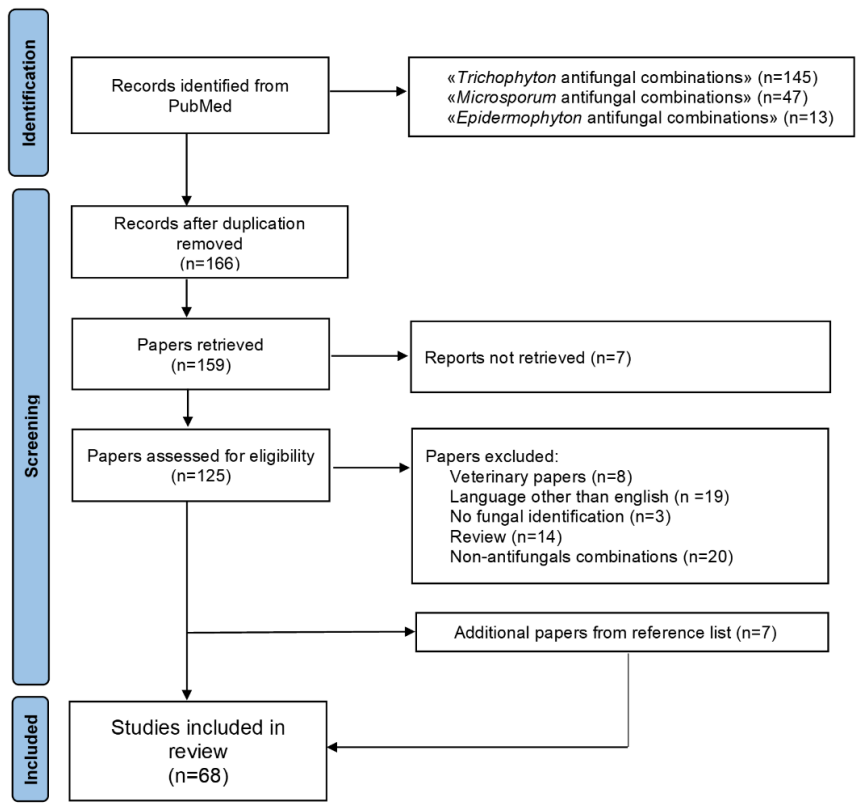

Figure 1. Flowchart of the different phases of article selection of the review.

\section{Results and Discussion}

A total of 205 articles were initially identified (Figure 1). After duplication removal, 166 articles were screened. Further exclusion included papers that were out of topic (34), veterinary (8), not in English (19), without fungal identification (3), literature reviews (14), and about combinations not including at least one antifungal agent (20). Additional 7 papers found in the reference list of the screened articles were added to the 61 eligible papers. Therefore, a total of 68 publications were included in this review: 37 articles referring to in vitro studies, and 31 articles referring to case reports or clinical studies (Tables 1-3). Among the first group of articles, there were 7 reports in which the combination of two antifungal agents was used, while 30 articles in which an antifungal agent was combined with a chemical compound other than an antifungal agent. 


\subsection{Antifungal Combinations}

The results of in vitro antifungal combinations are reported in Table 1. Trichophyton spp. represented the most common genus tested, followed by Microsporum spp. and E. floccosum. Combinations included amorolfine plus azoles or terbinafine or griseofulvin; azoles plus griseofulvin or terbinafine; azoles plus ciclopirox [24-30]. Checkerboard titration methodology was the most common procedure for testing a combination (6/7 studies). Two studies investigating the effects of the combination of amorolfine or ciclopirox plus azoles found $100 \%$ synergistic interaction against many Trichophyton spp. [26,28]. One study confirmed this positive effect by adding two additional methods (disk-diffusion and E-test assays) to the broth microdilution procedure [26]. Although antagonism was never observed in any report, the type of interaction varies according to drug and isolate tested. In general, amorolfine plus azoles yielded synergistic interaction more often than amorolfine plus griseofulvin or plus terbinafine. One study investigated three new topical drugs (efinaconazole, tavaborole, and luliconazole) with itraconazole or terbinafine against T. rubrum and T. interdigitale. Efinaconazole with terbinafine or itraconazole exerted a synergistic effect on $43.8 \%$ and $12.5 \%$ of the strains tested, respectively. Conversely, luliconazole showed no synergistic effect with terbinafine but was synergistically effective with itraconazole against $31.3 \%$ of the strains. Tavaborole (an inhibitor of protein synthesis in fungal cells) showed no synergistic effect with terbinafine and was synergistically effective with itraconazole against $18.8 \%$ of the strains [29].

Overall, these data would suggest that an antifungal combination regimen might be useful against an infection due to dermatophytes. It is interesting to note that even combining drugs acting against a common fungal target (i.e., ergosterol—azoles, allylamines, and morpholine drugs such as amorolfine), a positive interaction in terms of reduction of the MIC of both drugs is often observed.

\subsection{Antifungals Combined with Several Chemical Compounds}

The results of in vitro activities of antifungals combined with other compounds are reported in Table 2. Again, Trichophyton spp. represented the most common genus tested. Combination included: azoles or terbinafine or griseofulvin plus plant extracts including essential oils (19/30 studies), azoles or terbinafine or amorolfine plus immunosuppressant agents ( 3 studies), azoles or terbinafine plus peptides ( 3 studies), azoles plus disinfectants (3 studies), and other combinations including antifungal agents plus efflux pump inhibitors and statins. Checkerboard titration methodology was the most common procedure for testing the combination, followed by agar methods (i.e., disk diffusion and agar dilution) [31-60].

It has been shown that plants have the capacity to produce secondary metabolites, including those which are constituents of essential oils, as defence mechanisms against herbivores and microorganisms. They act in two ways: by neutralizing free radicals (the antioxidant effect) and as anti-inflammatory agents by inhibiting the release of proinflammatory mediators. Secondary metabolites produced by plants are also capable of acting in a third way, as antifungal agents [31-49]. A synergistic interaction between antifungal agents and natural products was often seen (Table 2). One recent study evaluated the antifungal activity of tea tree oil (TTO) (Melaleuca alternifolia essential oil) and its main components against T. rubrum alone and in association with ketoconazole or itraconazole and showed either their fungicidal effects or a synergism upon combination with azoles [49]. Most of the studies demonstrated that the type of interaction was either isolate- or drugdependent. One research assessed the antifungal activity of essential oil from Mentha $x$ piperita against a wide panel of dermatophyte clinical isolates and found a fungistatic activity against these fungi. When this compound was used in combination with azoles, a synergic interaction was observed for T. mentagrophytes while indifference was detected for T. rubrum and M. canis [48]. Overall, these data would suggest that these natural compounds are one of the most promising sources for pharmacological research and that 
the development of new natural antimicrobial agents against many microbial pathogens, including dermatophytes, is warranted.

Calcineurin inhibitors (i.e., tacrolimus and cyclosporin A) or inhibitors of the mTOR pathway (i.e., sirolimus) are anti-rejection drugs widely used in organ transplant recipients and to prevent graft-versus-host disease in allogeneic stem cell recipients. However, these compounds also possess intrinsic antifungal activity against selected fungi [50-52]. One study evaluated the in vitro interactions between tacrolimus or triamcinolone acetate with itraconazole, terbinafine, bifonazole, and amorolfine against 28 clinical dermatophyte isolates, including 13 T. rubrum, 6 T. mentagrophytes, 5 M. canis, and 4 E. floccosum and found that a synergistic interaction was more often observed when the antifungal agents were combined with tacrolimus rather than cortisone [52]. Another study evaluated the combination of fluconazole with either tacrolimus or cyclosporine in an ex vivo $T$. mentagrophytes human skin infection model. Conidia colonization was monitored by scanning electron microscopy over a 7-day treatment period. The fluconazole-tacrolimus combination was superior to one single-drug therapy by clearing conidia and protecting skin from damage at low drug concentrations [50]. Similarly, when tacrolimus was added to itraconazole against 5 isolates of $T$. mentagrophytes, a synergistic interaction was observed in $80 \%$ of the cases [51]. Overall, these data indicate that calcineurin inhibitors are synergistic with ergosterol biosynthesis inhibitors against dermatophytes, and that a potential clinical application may be desirable.

Table 1. Antifungal combinations against dermatophytes: in vitro effects of antifungal plus antifungal.

\begin{tabular}{|c|c|c|c|c|c|}
\hline Reference & $\begin{array}{c}\text { Number of Isolates and } \\
\text { Species }\end{array}$ & Combinations & Methods & Reading Endpoint & Results \\
\hline $\begin{array}{c}\text { Banic et al., } \\
1989 \text { [24] }\end{array}$ & $28 \mathrm{M}$. canis & GRI + KTZ & $\begin{array}{l}\text { Growth in Broth; } 28^{\circ} \mathrm{C}, \\
168 \mathrm{~h}\end{array}$ & $\%$ of inhibition & $\begin{array}{c}\text { Some strains of M. canis } \\
\text { were completely inhibited by } \\
\text { GRI + KTZ }\end{array}$ \\
\hline $\begin{array}{l}\text { Harman et al., } \\
2009 \text { [25] }\end{array}$ & $\begin{array}{c}\text { 4 T. rubrum, } \\
2 \text { T. mentag. var. interdigitale, } \\
2 \text { T. mentag. var. granulare, } \\
1 \text { T. tonsurans }\end{array}$ & $\mathrm{AMF}+\mathrm{TER} / \mathrm{FLU} / \mathrm{ITZ}$ & $\mathrm{Ck} ; 27^{\circ} \mathrm{C}, 168 \mathrm{~h}$ & $\geq 80 \%$ inhibition & Additivism or indifference \\
\hline $\begin{array}{l}\text { Laurent et al., } \\
2017 \text { [26] }\end{array}$ & 9 T. rubrum & $\begin{array}{c}\mathrm{AMF}+ \\
\mathrm{ITZ} / \mathrm{KTZ} / \mathrm{MIZ} / \mathrm{SER} / \mathrm{SUL}\end{array}$ & $\begin{array}{c}\text { Ck, disk diffusion and } \\
\text { E-test assay; } 30^{\circ} \mathrm{C} \\
168 \mathrm{~h}\end{array}$ & $\geq 80 \%$ inhibition & Synergy: $100 \%$ \\
\hline $\begin{array}{l}\text { Polak et al., } \\
1993 \text { [27] }\end{array}$ & $\begin{array}{l}3 \text { T. mentagrophytes, } \\
1 \text { T. rubrum, } 2 \text { M. canis, }\end{array}$ & $\begin{array}{c}\mathrm{AMF}+ \\
\mathrm{ITZ} / \mathrm{FLU} / \mathrm{GRI} / \mathrm{TER} / \mathrm{KET}\end{array}$ & $\begin{array}{c}\text { Agar dilution } \mathrm{Ck} ; 30^{\circ} \mathrm{C} \text {, } \\
96 \mathrm{~h}\end{array}$ & No visible growth & $\begin{array}{c}\text { Synergy: AMF + GRI 16\%; } \\
\text { AMF + KET 50\%; AMF + ITZ } \\
\text { 66\%; AMF + TER 50\%. } \\
\text { Indifference: } 100 \% \\
\text { AMF + FLU }\end{array}$ \\
\hline $\begin{array}{l}\text { Santos et al., } \\
2006 \text { [28] }\end{array}$ & $\begin{array}{l}52 \text { T. rubrum, } \\
40 \text { T. mentagrophytes }\end{array}$ & $\mathrm{CCL}+\mathrm{ITZ} / \mathrm{KTZ}$ & $\mathrm{Ck} ; 28^{\circ} \mathrm{C}, 168 \mathrm{~h}$ & $\geq 80 \%$ inhibition & Synergy: $100 \%$ \\
\hline $\begin{array}{l}\text { Sugiura et al., } \\
2021 \text { [29] }\end{array}$ & $\begin{array}{l}8 \text { T. rubrum, } \\
8 \text { T. interdigitale }\end{array}$ & $\begin{array}{c}\text { EFZ + TER, EFZ + ITZ, } \\
\text { LUZ + TER, LUZ + ITZ, } \\
\text { TAV + TER, TAV + ITZ, } \\
\text { LUZ + TAV }\end{array}$ & $\mathrm{Ck}, 35^{\circ} \mathrm{C}, 96 \mathrm{~h}$ & $\geq 80 \%$ inhibition & $\begin{array}{c}\text { Synergy: EFZ + TER 43.8\%, } \\
\text { EFZ + ITZ 12.5\%, LUZ + ITZ } \\
\text { 31.25\%, TAV + ITZ 18.7\%. } \\
\text { Additivism: EFZ + TER } \\
43.75 \% \text {, EFZ + ITZ } 18.75 \% \text {, } \\
\text { LUZ + TER 31.25\%, LUZ + } \\
\text { ITZ 18.75\%, TAV + TER 25\%, } \\
\text { TAV + ITZ 6.25\%. } \\
\text { Indifference: EFZ + TER } \\
\text { 12.5\%, EFZ + ITZ 68.75\%, } \\
\text { LUZ + TER 68.75\%, LUZ + } \\
\text { ITZ 50\%, TAV + ITZ/TER } \\
\text { 75\%. LUZ + TAV indifferent } \\
\text { effect on some strain }\end{array}$ \\
\hline $\begin{array}{l}\text { Tamura et al., } \\
2014[30]\end{array}$ & $\begin{array}{l}11 \text { T. rubrum, } \\
8 \text { T. Mentagrophytes, } \\
1 \text { T. tonsurans, } \\
1 \text { T. verrucosum, } \\
\text { 3 M. gypseum, } \\
\text { 3 E. floccosum }\end{array}$ & $\mathrm{AMF}+\mathrm{ITZ}$ & $\mathrm{Ck} ; 30^{\circ} \mathrm{C}, 72-168 \mathrm{~h}$ & $\geq 80 \%$ inhibition & $\begin{array}{c}\text { Synergistic interactions: } \\
25.9 \% \\
\text { Additivism interactions: } \\
59.2 \% \text {. } \\
\text { Indifference effect: } 14.9 \% \\
\text { No antagonistic effects } \\
\text { were detected }\end{array}$ \\
\hline
\end{tabular}

GRI, griseofulvin; KTZ, ketoconazole; AMF, amorolfine; TER, terbinafine; FLU, fluconazole; ITZ, itraconazole; MIZ, miconazole; SER, sertaconazole; SUL, sulconazole; CCL, cyclopirox; EFZ, efinaconazole; LUZ, luliconazole; TAV, tavaborole; Ck, Checkerboard titration (performed in broth or otherwise specified). M., Microsporum; T., Trichophyton; E., Epidermophyton. The interaction was defined as synergistic if the FIC index (FICI) was $\leq 0.5$, additivism if $>0.50$ but $<1.0$, indifferent if FICI was $\geq 1$ but $\leq 4.0$, and antagonistic if FICI was $>4.0$. 
Table 2. Antifungal combinations against dermatophytes: in vitro effects of an antifungal plus a chemical compound other than antifungal.

\begin{tabular}{|c|c|c|c|c|c|}
\hline Reference & $\begin{array}{l}\text { Number of Isolates and } \\
\text { Species }\end{array}$ & Combinations & Methods & Reading Endpoint & Results \\
\hline $\begin{array}{l}\text { Danielli et al., } \\
2018 \text { [31] }\end{array}$ & $\begin{array}{l}2 \text { T. rubrum, } \\
2 \text { T. mentagrophytes, } \\
2 \text { M. canis, } \\
2 \text { M. gypseum }\end{array}$ & $\begin{array}{l}\text { Schinus lentiscifolius } \\
\text { Marchand + TER/CCL }\end{array}$ & Ck, time-kill curves; & $100 \%$ inhibition & $\begin{array}{l}\text { Synergy: EO + TER } 50 \% \text {, EO } \\
\text { + CCL } 25 \% \text {. Additivism: EO } \\
\text { + TER 37.5\%, EO + CCL } \\
\text { 62.5\%. Indifference: EO + } \\
\text { TER } 12.5 \% \text {, EO + CCL } 12.5 .\end{array}$ \\
\hline $\begin{array}{l}\text { Dias et al., } \\
2017 \text { [32] }\end{array}$ & $\begin{array}{l}1 \text { T. rubrum, } \\
1 \text { T. mentagrophytes }\end{array}$ & $\begin{array}{l}\text { E.O. L. lusieri/E.O. } \\
\text { C. citratus + TER }\end{array}$ & $\begin{array}{l}\text { Fixed ratio combination; } \\
\qquad 30^{\circ} \mathrm{C}, 96 \mathrm{~h}\end{array}$ & $\geq 90 \%$ inhibition & $\begin{array}{l}5 \% \text { Growth in } 1: 1 \\
\text { combination EO L. lusieri + } \\
\text { TER, } 20 \% \text { growth in } 1: 1 \\
\text { combination EO } \\
\text { C. citratus + TER }\end{array}$ \\
\hline $\begin{array}{l}\text { Ala et al., } 2010 \\
{[33]}\end{array}$ & $\begin{array}{l}1 \text { T. rubrum, } \\
1 \text { T. mentagrophytes, } \\
1 \text { T. verrucosum, } \\
1 \text { E. flocossum }\end{array}$ & Allicin + KTZ/FLU & $\mathrm{Ck} ; 28^{\circ} \mathrm{C}, 168-240 \mathrm{~h}$ & $\geq 50-90 \%$ inhibition & $\begin{array}{c}\text { Synergy/additivism: } 54 \% \text {, } \\
\text { indifference: } 46 \% \text { after } \\
7 \text { days. Synergy/additivism: } \\
33.5 \%, 66.5 \% \text {. } \\
\text { Indifference: after } 10 \text { days. }\end{array}$ \\
\hline $\begin{array}{l}\text { Galgóczy et al., } \\
2008[34]\end{array}$ & $\begin{array}{c}2 \text { M. canis, } \\
1 \text { M. gypseum, } \\
3 \text { T. mentagrophytes, } \\
1 \text { T. rubrum, } \\
1 \text { T. tonsurans }\end{array}$ & $\begin{array}{c}\text { PAF (Penicillin } \\
\text { Chrysogeneum } \\
\text { Antifungal Protein) } \\
\quad+\text { FLU }\end{array}$ & $\mathrm{Ck} ; 37^{\circ} \mathrm{C}, 96-168-240 \mathrm{~h}$ & $\%$ of inhibition & $\begin{array}{l}\text { Decreased growth when } \\
\text { used in combination }\end{array}$ \\
\hline $\begin{array}{l}\text { Houël et al., } \\
2014 \text { [35] }\end{array}$ & $\begin{array}{l}1 \text { T. mentagrophytes, } \\
1 \text { M. gypseum }\end{array}$ & $\begin{array}{l}\text { E.O. Otacanthus azureus } \\
+ \text { ITZ/FLU/KTZ }\end{array}$ & $\mathrm{Ck} ; 32{ }^{\circ} \mathrm{C}, 120 \mathrm{~h}$ & No visible growth & $\begin{array}{l}\text { Synergy in T. mentagrophytes, } \\
\text { indifference in M. gypseum }\end{array}$ \\
\hline $\begin{array}{l}\text { Khan et al., } \\
2011[36]\end{array}$ & 1 T. rubrum & $\begin{array}{c}\text { S. aro- } \\
\text { maticum/eugenol/C. } \\
\text { verum/cinnamaldehyde/C. } \\
\text { martini/geraniol + FLU }\end{array}$ & $\mathrm{Ck} ; 30^{\circ} \mathrm{C}, 48 \mathrm{~h}$ & No visible growth & $\begin{array}{l}\text { Synergy: } 100 \% \text { in all } \\
\text { combinations }\end{array}$ \\
\hline $\begin{array}{l}\text { Khan et al., } \\
2014 \text { [37] }\end{array}$ & 1 T. rubrum & $\begin{array}{l}\text { E.O. C. copticum or E.O. } \\
\text { T. vulgaris or thymol } \\
+ \text { FLU }\end{array}$ & $\mathrm{Ck} ; 30^{\circ} \mathrm{C}, 48 \mathrm{~h}$ & No visible growth & $\begin{array}{l}\text { Synergy: E.O. T. vulgaris or } \\
\text { thymol + FLU. Indifference: } \\
\text { C. copticum + FLU }\end{array}$ \\
\hline $\begin{array}{l}\text { Khoury et al. } \\
\text { 2019, [38] }\end{array}$ & $\begin{array}{l}1 \text { T. rubrum, } \\
1 \text { T. mentagrophytes, } \\
1 \text { T. violaceum, } \\
1 \text { T. soudanense, } \\
1 \text { T. tonsurans }\end{array}$ & $\begin{array}{l}\text { E.O. Hitellina lobelii }+ \\
\text { FLU/GRI }\end{array}$ & $\mathrm{Ck} ; 25^{\circ} \mathrm{C}, 72 \mathrm{~h}$ & No visible growth & $\begin{array}{c}\text { Synergy in all strains, except } \\
\text { for additivity EO + FLU in } \\
\text { T. tonsurans }\end{array}$ \\
\hline $\begin{array}{l}\text { Maciel et al., } \\
2019 \text { [39] }\end{array}$ & $\begin{array}{l}\text { 3T. mentagrophytes, } \\
2 \text { T. rubrum, } \\
1 \mathrm{M} \text { gypseum }\end{array}$ & $\begin{array}{l}\text { E.O. Cryptocarya } \\
\text { aschersoniana + TER }\end{array}$ & $\mathrm{Ck} ; 35^{\circ} \mathrm{C}, 48 \mathrm{~h}$ & No visible growth & $\begin{array}{l}\text { Indifference for all strains } \\
\text { except for additivism in } 1 . \\
\text { T. rubrum }\end{array}$ \\
\hline $\begin{array}{l}\text { Pyun et al., } \\
2005 \text { [40] }\end{array}$ & $\begin{array}{l}1 \text { T. rubrum, } \\
1 \text { T. erinacei, } \\
1 \text { T. soudanense }\end{array}$ & $\begin{array}{l}\text { Allium sativum/Allicin } \\
+\mathrm{KTZ}\end{array}$ & $\mathrm{Ck} ; 24-28{ }^{\circ} \mathrm{C}, 72 \mathrm{~h}$ & No visible growth & $\begin{array}{c}\text { Synergy: A. sativum + } \\
\text { KTZ 100\%. } \\
\text { Additivism: Allicin + } \\
\text { KTZ } 100 \%\end{array}$ \\
\hline $\begin{array}{l}\text { Roana et al., } \\
2021[41]\end{array}$ & 1 T. rubrum & $\begin{array}{c}\text { Tea tree oil }(\mathrm{TTO})+ \\
\text { ITZ/KTZ }\end{array}$ & $\mathrm{Ck} ; 28-30{ }^{\circ} \mathrm{C}, 168 \mathrm{~h}$ & No visible growth & $\begin{array}{l}\text { Synergy with both } \\
\text { combinations }\end{array}$ \\
\hline $\begin{array}{c}\text { Rodriguez } \\
\text { et al., } 2013 \text { [42] }\end{array}$ & 1 T. rubrum & $\begin{array}{l}44 \text { extracts from } 9 \\
\text { Baccharis spp. And } 4 \\
\text { flavonoids and } 3 \\
\text { ent-clerodanes + TER }\end{array}$ & $\begin{array}{c}\text { HTSS assay, fixed } \\
\text { concentration; } 28-30{ }^{\circ} \mathrm{C}, \\
168 \mathrm{~h}\end{array}$ & No visible growth & $\begin{array}{c}\text { Synergy with bacrispine or } \\
\text { baccho A + TER }\end{array}$ \\
\hline $\begin{array}{l}\text { Shin et al., } \\
2004 \text { [43] }\end{array}$ & $\begin{array}{l}1 \text { T. erinacei, } \\
1 \text { T. mentagrophytes, } \\
1 \text { T. rubrum, } \\
1 \text { T. tonsurans, } \\
1 \text { T. schoenleinii, } \\
1 \text { T. soudanense }\end{array}$ & $\begin{array}{c}\text { P. graveolens oil, } \\
\text { citronellol, and geraniol } \\
+ \text { KTZ }\end{array}$ & $\mathrm{Ck} ; 25^{\circ} \mathrm{C}, 72 \mathrm{~h}$ & No visible growth & Synergy: $100 \%$ \\
\hline $\begin{array}{l}\text { Shin et al., } \\
2004[44]\end{array}$ & $\begin{array}{l}1 \text { T. erinacei, } \\
1 \text { T. mentagrophytes, } \\
1 \text { T. rubrum, } \\
1 \text { T. schoenleinii, } \\
1 \text { T. soudanense. }\end{array}$ & $\begin{array}{l}\text { E.O. fraction of } \\
\text { A. rugosa + KTZ }\end{array}$ & $\mathrm{Ck} ; 25^{\circ} \mathrm{C}, 72 \mathrm{~h}$ & No visible growth & Synergy: $100 \%$ \\
\hline $\begin{array}{c}\text { Sim et al., } 2008 \\
{[45]}\end{array}$ & $\begin{array}{l}1 \text { T. erinacei, } \\
1 \text { T. mentagrophytes, } \\
1 \text { T. rubrum, } \\
1 \text { T. schoenleinii, } \\
1 \text { T. soudanense, } \\
1 \text { T. tonsurans }\end{array}$ & $\begin{array}{l}\text { Ligustilide/Butylidene } \\
\text { phthalide + ITZ/KTZ }\end{array}$ & $\mathrm{Ck} ; 25^{\circ} \mathrm{C}, 72 \mathrm{~h}$ & $\geq 50 \%$ inhibition & $\begin{array}{c}\text { Synergy: } 35 \% \\
\text { Additivism: } 65 \%\end{array}$ \\
\hline $\begin{array}{l}\text { Soares et al., } \\
2014 \text { [46] }\end{array}$ & $\begin{array}{l}3 \text { T. rubrum, } \\
3 \text { T. mentagrophytes }\end{array}$ & $\begin{array}{l}\text { Protocatechuic acids } \\
\qquad(\mathrm{n}=5)+\text { FLU }\end{array}$ & $\mathrm{Ck} ; 35^{\circ} \mathrm{C}, 168 \mathrm{~h}$ & $\geq 50 \%$ inhibition & $\begin{array}{l}\text { Synergy: } 1 \text { T. mentragrophytes } \\
\text { PA9 + FLU. Additivism or } \\
\text { indifference in other cases. }\end{array}$ \\
\hline $\begin{array}{l}\text { Tiwari et al., } \\
2017 \text { [47] }\end{array}$ & $\begin{array}{l}1 \text { T. mentagrophytes, } \\
1 \mathrm{M} \text {. canis }\end{array}$ & $\begin{array}{l}\mathrm{ZnO} \text { particles from } \\
\text { Rosa indaca }+\mathrm{KTZ}\end{array}$ & $\begin{array}{l}\text { Disk diffusion; } 28^{\circ} \mathrm{C}, \\
48 \mathrm{~h}\end{array}$ & Inhibition diameter & $\begin{array}{l}\text { Decreased growth when } \\
\text { used in combination }\end{array}$ \\
\hline
\end{tabular}


Table 2. Cont.

\begin{tabular}{|c|c|c|c|c|c|}
\hline Reference & $\begin{array}{c}\text { Number of Isolates and } \\
\text { Species }\end{array}$ & Combinations & Methods & Reading Endpoint & Results \\
\hline $\begin{array}{l}\text { Tullio et al., } \\
2019 \text { [48] }\end{array}$ & $\begin{array}{l}\text { 1T. mentagrophytes, } \\
1 \mathrm{M} \text {. canis, } \\
1 \mathrm{~T} \text {. rubrum }\end{array}$ & $\begin{array}{c}\text { E.O. Menta piperita }+ \\
\text { ITZ/KTZ }\end{array}$ & $\mathrm{Ck} ; 30^{\circ} \mathrm{C}, 168 \mathrm{~h}$ & No visible growth & $\begin{array}{c}\text { Synergy in T. mentagrophytes, } \\
\text { indifference in M. canis and } \\
\text { T. rubrum }\end{array}$ \\
\hline $\begin{array}{l}\text { Vörös- } \\
\text { Horváth et al., } \\
2020[49]\end{array}$ & 1 T. rubrum & $\begin{array}{l}\text { E.O. Melaleuca } \\
\text { altifornia + TIO }\end{array}$ & $\mathrm{Ck} ; 28^{\circ} \mathrm{C}, 168 \mathrm{~h}$ & No visible growth & Synergy: $100 \%$ \\
\hline $\begin{array}{l}\text { Onyewu et al., } \\
2007 \text { [50] }\end{array}$ & 2 T. mentagrophytes & $\begin{array}{l}\text { cyclosporine A or } \\
\text { FK506 + FLU }\end{array}$ & $\begin{array}{c}\text { Ck + ex vivo } T . \\
\text { mentagrophytes human } \\
\text { skin infection model }\end{array}$ & $\geq 80 \%$ inhibition & $\begin{array}{c}\text { Synergy in all cases except } \\
\text { indifference FKS506+FLU } \\
\text { against } 1 \text { strain }\end{array}$ \\
\hline $\begin{array}{l}\text { Ozawa et al., } \\
2005[51]\end{array}$ & 5 T. mentagrophytes & $\mathrm{TAC}+\mathrm{ITR}$ & $\begin{array}{l}\text { Agar dilution, } \mathrm{Ck} ; \\
27^{\circ} \mathrm{C}, 168 \mathrm{~h}\end{array}$ & $\geq 50 \%$ inhibition & Synergy: $80 \%$ \\
\hline $\begin{array}{l}\text { Zhang et al., } \\
2018 \text { [52] }\end{array}$ & $\begin{array}{l}13 \text { T. rubrum, } \\
\text { 6 T. mentagrophytes, } \\
5 \text { M. canis, } \\
4 \text { E. floccosum }\end{array}$ & $\begin{array}{c}\text { TAC/TRI + } \\
\text { ITZ/TER/BIZ/AMF }\end{array}$ & $\mathrm{Ck} ; 35^{\circ} \mathrm{C}, 96-120 \mathrm{~h}$ & $\geq 80-100 \%$ inhibition & $\begin{array}{l}\text { Synergy: TAC/ITZ 39\%, } \\
\text { TAC/TRB } 43 \% \text {, TAC/BIZ } \\
\text { 43\%, TRI/ITZ 7\%, TRI/BIZ } \\
\text { 11\%. Indifference in all } \\
\text { other cases. }\end{array}$ \\
\hline $\begin{array}{l}\text { Simonetti } \\
\text { et al., } 2009 \text { [53] }\end{array}$ & $\begin{array}{l}\text { 6 M. canis, } \\
\text { 6 T. mentagrophytes, } \\
10 \text { T. rubrum, } \\
2 \text { M. gypseum }\end{array}$ & $\begin{array}{c}\text { lipopeptide } \\
\text { Pal-Lys-Lys-NH2 (PAL) } \\
\text { + FLU/ITZ/TER }\end{array}$ & $\mathrm{Ck} ; 35^{\circ} \mathrm{C}, 96 \mathrm{~h}$ & $\geq 90 \%$ inhibition & $\begin{array}{c}\text { Synergy: PAL/TER 52\%, } \\
\text { PAL/ITZ 67\%, } \\
\text { PAL/FLU15\%. } \\
\text { Indifference: PAL/TER 48\%, } \\
\text { PAL/ITZ 33\%, } \\
\text { PAL/FLU 85\% }\end{array}$ \\
\hline $\begin{array}{l}\text { Simonetti } \\
\text { et al., } 2009 \text { [54] }\end{array}$ & $\begin{array}{l}4 \text { M. canis, } \\
5 \text { T. mentagrophytes, } \\
9 \text { T. rubrum, } \\
2 \text { M. gypseum }\end{array}$ & Tachiplesina III + TER & $\begin{array}{c}\mathrm{Ck} ; 35^{\circ} \mathrm{C} \text {, until visible } \\
\text { growth }\end{array}$ & $\geq 90 \%$ inhibition & $\begin{array}{c}\text { Synergy: } 30 \% \\
\text { Indifference: } 70 \%\end{array}$ \\
\hline $\begin{array}{l}\text { Simonetti } \\
\text { et al., } 2014 \text { [55] }\end{array}$ & $\begin{array}{l}6 \text { M. canis, } \\
\text { 6 T. mentagrophytes, } \\
8 \text { T. rubrum }\end{array}$ & IB-367 + TER/FLU/ITZ & $\begin{array}{l}\mathrm{Ck} \text {, time-kill curves; } \\
35^{\circ} \mathrm{C} \text {, until visible } \\
\text { growth }\end{array}$ & No visible growth & $\begin{array}{c}\text { Synergy: M. canis IB-367 + } \\
\text { FLU 50\%, IB-367 + ITZ 17\%, } \\
\text { IB-367 + TER 33\%; T. } \\
\text { mentagrophytes IB-367 + FLU } \\
\text { 33\%, IB-367 + ITZ 67\%, } \\
\text { IB-367 + TER 17\%; T. rubrum } \\
\text { IB-367 + FLU 25\%, IB-367 + } \\
\text { ITZ 13\%, IB-367 + TER 25\% }\end{array}$ \\
\hline $\begin{array}{l}\text { Moriello et al., } \\
2007 \text { [56] }\end{array}$ & 1 M. canis & $\mathrm{CLO}+\mathrm{MIZ}$ & Growth in broth & No visible growth & No growth \\
\hline $\begin{array}{l}\text { Perrins N., } \\
\text { et al. } 2003 \text { [57] }\end{array}$ & 10 M. canis & $\mathrm{CLO}+\mathrm{MIZ}$ & $\begin{array}{l}\text { Agar Dilution: } 26^{\circ} \mathrm{C} \text {, } \\
\qquad 120 \mathrm{~h}\end{array}$ & No visible growth & $\begin{array}{c}\text { Synergy: } 50 \% \\
\text { Additivism: } 40 \% \\
\text { Indifference: } 10 \%\end{array}$ \\
\hline $\begin{array}{l}\text { Perrins et al., } \\
2005[58]\end{array}$ & $\begin{array}{l}9 \text { T. mentagrophytes, } \\
9 \text { T. erinacei, } \\
5 \text { M. persicolor }\end{array}$ & CLO + MIZ & $\begin{array}{c}\text { Agar dilution; } 26^{\circ} \mathrm{C}, \\
168 \mathrm{~h}\end{array}$ & No visible growth & $\begin{array}{c}\text { Synergy: } 8.70 \% \\
\text { Additivism: } 56.52 \% \\
\text { Indifference: } 34.78\end{array}$ \\
\hline $\begin{array}{l}\text { Nyilasi et al., } \\
2014 \text { [59] }\end{array}$ & $\begin{array}{l}1 \text { T. rubrum, } \\
1 \text { T. mentagrophytes, } \\
1 \text { M. gypseum, } \\
1 \text { M. canis }\end{array}$ & $\begin{array}{c}\mathrm{LOV} / \mathrm{SIM} / \mathrm{FLV} / \mathrm{ROS} / \\
\mathrm{ATO} / \mathrm{PRA} / \mathrm{NYT} / \mathrm{PN}+ \\
\mathrm{AMB} / \mathrm{KTZ} / \mathrm{ITZ} / \\
\text { FLU/TER/GRI }\end{array}$ & $\mathrm{Ck} ; 30^{\circ} \mathrm{C}, 96 \mathrm{~h}$ & No visible growth & $\begin{array}{c}\text { Synergy: } 85.92 \% \\
\text { Indifference: } 14.08 \%\end{array}$ \\
\hline $\begin{array}{l}\text { Aneke et al., } \\
2020 \text { [60] }\end{array}$ & 36 M. canis & $\begin{array}{l}\text { Haloperidol/ } \\
\text { promethazine + } \\
\text { ITZ/FLU }\end{array}$ & $\begin{array}{c}\mathrm{Ck} \text {, disk diffusion, } \\
\text { time-kill curve; } 30^{\circ} \mathrm{C}, \\
48 \mathrm{~h}\end{array}$ & $\geq 80 \%$ inhibition & $\begin{array}{c}\text { Synergy: ITZ + PRO 91.7\%, } \\
\text { ITZ + HAL 77.8\%, FLU + } \\
\text { PRO 25\%, FLU + HAL 5.5\%. } \\
\text { Indifference: ITZ + PRO } \\
\text { 8.3\%, ITZ + HAL 22.2\%, FLU } \\
\text { + PRO 47.2\%, } \\
\text { FLU + HAL } 61.2 \% . \\
\text { Antagonism: FLU + PRO } \\
\text { 27.8\%, FLU + HAL } 33.1 \% .\end{array}$ \\
\hline
\end{tabular}

TER, terbinafine; CCL, ciclopirox; KTZ, ketoconazole; FLU, fluconazole; ITZ, itraconazole; GRI, griseofulvin; TAC, tacrolimus; TRI, triamcinolone acetonide, BIZ, bifonazole; AMF, amorolfine; CLO, chlorhexidine; MIZ, miconazole; LOZ, lovastatin; SIM, simvastatin; FLV, fluvastatin; ROS, rosuvastatin; ATO, atorvastatin; PRA, pravastatin; NYT, nystatin; PN, prymicin. Ck, Checkerboard titration (performed in broth or otherwise specified). M., Microsporum; T., Trichophyton; E., Epidermophyton. E.O., essential oil. The interaction was defined as synergistic if the FIC index (FICI) was $\leq 0.5$, additivism if $>0.50$ but $<1.0$, indifferent if FICI was $\geq 1$ but $\leq 4.0$, and antagonistic if FICI was $>4.0$.

Another interesting therapeutic approach might be represented by peptides because they act efficiently and rapidly against a wide range of pathogens including bacteria, fungi, viruses, and protozoa. Moreover, peptide resistant mutants rarely emerge with these molecules, especially when they are used in combination with other anti-infective drugs [53-55]. Among these compounds, protegrins and defensin were originally iso- 
lated from mammalian leucocytes. One study evaluated the in vitro effects of IB-367 alone and in combination with three antifungal drugs against 20 clinical isolates of dermatophytes belonging to three species and showed synergism in $35 \%, 30 \%$, and $25 \%$ of IB-367/fluconazole, IB-367/itraconazole, and IB-367/terbinafine interactions, respectively. IB-367 exerted a fungicidal activity against T. mentagrophytes, T. rubrum, and M. canis at concentrations starting from $1 \times$ MIC. At a concentration of $5 \times$ MIC, IB-367 showed the highest rates of hyphae damage for M. canis and T. mentagrophytes [55]. Another study investigated the in vitro effects of tachyplesin III (TP), a potent disulphide-linked peptide, in combination with terbinafine against 20 clinical isolates of dermatophytes belonging to four species. Terbinafine in combination with TP showed indifferent activity against 14 of the 20 isolates $(70 \%)$; synergic activity against 6 of them (30\%); no antagonistic activity was observed [54]. Finally, the lipopeptide Pal-Lys-Lys- $\mathrm{NH}_{2}$ (PAL) alone and in combination with standard antifungal agents was tested against 24 clinical isolates of dermatophytes belonging to four species. Synergy was observed in $67 \%, 52 \%$, and $15 \%$ of PAL/itraconazole, $\mathrm{PAL} /$ terbinafine, and PAL/fluconazole interactions, respectively. None of these combinations yielded antagonistic interactions. When synergy was not achieved, there was still a decrease in the MIC of one or both drugs used in the combination [53]. Overall, these studies demonstrated that peptides have potential activity against dermatophytes. These drugs, applied in the form of lacquer, spray, or ointment, could represent an interesting new therapy, particularly when combined with conventional treatment in recalcitrant or resistant dermatophyte infections.

Another combinatorial approach investigated the activity of an antifungal, generally miconazole, with the antiseptic compound chlorhexidine [56-58]. One study demonstrated that this association yielded a synergistic effect in vitro against 5 out of 10 isolates of $M$. canis, and an additive effect against 4 isolates, while when the same combination was tested against 9 isolates, each of $T$. mentagrophytes and T. erinacei, the most frequent interactions observed were additivism or indifference. Again, antagonism was never observed [57,58].

In general, the results obtained by combination of antifungal agents with chemical compounds other than antifungals yielded variable results spanning from synergism to indifference. Antagonism was rarely seen. This interaction is well documented for natural products (i.e., essential oils) as shown by a substantial number of scientific publications. Although promising results were documented, the different methods used to test these combinations hampered a univocal and comprehensive conclusion on the real effectiveness of these combinations.

\subsection{Clinical Cases}

The results of antifungal combinations in humans are reported in Table 3. There were 25 papers describing 37 single case reports, one paper each describing 36 and 254 patients, respectively, and 3 clinical trials involving a total of 410 patients [61-91]. Either paediatric or adult patients were represented. Tinea corporis, tinea capitis, and tinea unguium were the most common clinical manifestations. In the single case reports, the most frequent combination approach included a systemic antifungal agent administered orally (i.e., terbinafine, griseofulvin, or azole-mainly itraconazole) plus a topical medication (i.e., azole, terbinafine, ciclopirox, cortisone) for several weeks. Few cases were treated with both drugs given topically or orally. Only in two clinical cases, resistance mechanisms were assessed and confirmed by sequencing of the SQLE gene [67,70]. One patient with Trichophyton endophthalmitis and five patients with fungal keratitis due to T. shoenleinii were treated with a combination of systemic antifungal agents, including voriconazole or fluconazole, plus an antifungal agent administered topically (amphotericin B or miconazole). The outcome consisted in full recovery or improvement in most of the cases $[74,86]$. The 36 patients included in one paper consisted of 18 children and 18 adults with infections due to T. violaceum. The source of contagion was traced to 13 children, 11 African and 2 Ukrainian, adopted from an orphanage, with misdiagnosed tinea capitis. All 13 index cases and the 16 patients infected by them were treated with griseofulvin for 45 days and 
topical imidazoles. The adults with spreading tinea corporis were treated with $100 \mathrm{mg}$ itraconazole for 15-20 days and those with tinea capitis with the same dose of the antimycotic for 45 days and with topical imidazoles. In all patients, recovery was confirmed by clinical and mycological examination 3 months after healing [87]. One early observational study involving 254 patients with various forms of dermatophyte infections mainly due to Trichophyton spp., concluded that topical treatment (Wilkinson's salve, iodized alcohol 5\%, undecylenic acid derivatives, 5-bromo-4'-chlorosalicylanilides, tolnaftates) plus griseofulvin possibly enhances the healing capacity and shortens the time for treatment, but it has no effect in preventing reinfections [88]. One randomized study of toenail onychomycosis with matrix area involvement due to T. rubrum in most cases, compared amorolfine $5 \%$ nail lacquer once weekly for 24 weeks given with $200 \mathrm{mg}$ of itraconazole once daily for 6 or 12 weeks vs. itraconazole alone given for 12 weeks [91]. Combination therapy showed to be significantly more effective than monotherapy, both in terms of mycological and clinical cures at week 12. Similarly, another randomized study comparing amorolfine plus terbinafine vs. terbinafine alone in 249 patients with onychomycosis showed a significantly higher success rate for patients undergoing combination therapy relative to those in monotherapy at 18 months [89]. Another randomized study investigated the efficacy of combination therapy with oral griseofulvin and oral prednisolone to oral griseofulvin alone in the treatment of kerion celsi due to Trichophyton spp. [91]. Both groups were treated with oral griseofulvin for 8 weeks, whereas oral prednisolone was given in tapering doses for 3-4 weeks to the first group only. The final evaluation at week 12 showed a cure rate of $100 \%$ in both groups without any significant difference in terms of clinical or mycological cure.

Table 3. Antifungal combinations against dermatophytes: clinical cases.

\begin{tabular}{|c|c|c|c|}
\hline Reference & Number of Isolates and Species & Combinations & Results \\
\hline $\begin{array}{l}\text { Adamski et al., } 2014 \\
\text { [61] }\end{array}$ & $\begin{array}{l}\text { A 34-year-old Polish Caucasian } \\
\text { male with erythematous, } \\
\text { exfoliating, clearly distinct lesion } \\
\text { located on the index finger of the } \\
\text { right hand caused by T. rubrum }\end{array}$ & $\begin{array}{l}\text { ITZ daily dose } 100 \mathrm{mg} \text { and topical IMZ at } \\
\text { first; subsequently the topical drug was } \\
\text { switched to a pyridinone derivative }\end{array}$ & Full recovery \\
\hline $\begin{array}{l}\text { Budihardja et al., } \\
\quad 2010[62]\end{array}$ & $\begin{array}{l}\text { 45-year-old patient, renal } \\
\text { transplant recipient with } \\
\text { widespread erosive tinea corporis } \\
\text { caused by T. mentagrophytes }\end{array}$ & $\begin{array}{l}\text { TER daily plus CCL olamine topically for } \\
9 \text { weeks }\end{array}$ & Clinical cure \\
\hline $\begin{array}{l}\text { Czaika et al., } 2013 \\
\text { [63] }\end{array}$ & $\begin{array}{l}\text { Two girls (11 and } 7 \text { years) with } \\
\text { zoophile tinea faciei and tinea } \\
\text { corporis due to T. mentagrophytes }\end{array}$ & $\begin{array}{l}\text { Systemic TER at a daily dose of } 125 \mathrm{mg} \text {, } \\
\text { based on body weight for } 5 \text { weeks } \\
\text { (11-year-old girl) and for } 4 \text { weeks } \\
\text { (7-year-old girl) was prescribed. Twice } \\
\text { daily, application of ISZ/DFV cream } \\
\text { containing ISN } 1 \% \text { and DFV } 0.1 \% \text { was } \\
\text { prescribed for } 10 \text { days (facial lesion) or } \\
14 \text { days (other lesions), subsequently to be } \\
\text { continued with CCL. }\end{array}$ & $\begin{array}{l}\text { Improvement of all } \\
\text { lesions and pruritus in } \\
\text { both patients } 2 \text { weeks } \\
\text { after treatment } \\
\text { initiation }\end{array}$ \\
\hline $\begin{array}{l}\text { Durant et al., } 2009 \\
{[64]}\end{array}$ & $\begin{array}{l}\text { A 31-year-old patient presented } \\
\text { with a diagnosis of } \\
\text { granulomatous dermatophytosis } \\
\text { due to T. rubrum }\end{array}$ & ITZ plus TER $250 \mathrm{mg}$ & No improvement \\
\hline $\begin{array}{l}\text { Fabrizi et al., } 2017 \\
\text { [65] }\end{array}$ & $\begin{array}{l}\text { A 74-years-old with interdigital } \\
\text { tinea pedis and distal-lateral } \\
\text { onychomycosis of both big toes } \\
\text { were present due to T. rubrum and } \\
\text { Tyrophagus putrescentiae }\end{array}$ & $\begin{array}{c}\text { TER } 250 \mathrm{mg} / \text { day and CCL } 8 \% \text { nail lacquer } \\
\text { for } 16 \text { weeks }\end{array}$ & Full recovery \\
\hline $\begin{array}{l}\text { Ghislanzoni, } 2008 \\
\text { [66] }\end{array}$ & $\begin{array}{l}\text { A 35-year-old male with tinea } \\
\text { incognito due to T. rubrum }\end{array}$ & Topic ISZ plus DFC for 4 weeks & Partial improvement \\
\hline
\end{tabular}


Table 3. Cont

\begin{tabular}{|c|c|c|c|}
\hline Reference & Number of Isolates and Species & Combinations & Results \\
\hline Hsieh et al., 2019 [67] & $\begin{array}{l}\text { A } 60 \text {-year-old man and a } \\
\text { 51-year-old-woman with } \\
\text { disseminated tinea corporis } \\
\text { caused by T. mentagrophytes }\end{array}$ & ITZ with topical EBE & Full Recovery \\
\hline Jang et al., 2017 [68] & $\begin{array}{l}\text { A 9-year-old male with kerion } \\
\text { celsi caused by T. erinacei }\end{array}$ & $\begin{array}{l}\text { TER } 250 \mathrm{mg} \text { / day for } 6 \text { weeks and MTP } \\
12 \mathrm{mg} \text { /day for the first week. }\end{array}$ & Full recovery \\
\hline $\begin{array}{l}\text { Khaled et al., } 2007 \\
\text { [69] }\end{array}$ & $\begin{array}{l}\text { A 6-year-old Tunisian boy with } \\
\text { tinea favosa due to T. schoenleinii }\end{array}$ & $\begin{array}{l}20 \mathrm{mg} / \mathrm{kg} / \text { day of oral GRI } 400 \mathrm{mg} \\
\text { twice daily for } 6 \text { weeks and topical IMZ } \\
\text { for } 8 \text { weeks }\end{array}$ & Full recovery \\
\hline $\begin{array}{l}\text { Kimura et al., } 2020 \\
\text { [70] }\end{array}$ & 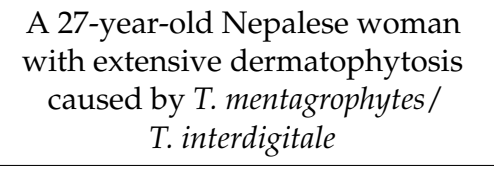 & Oral ITZ 100 mg/day and topical LUZ & Full recovery \\
\hline $\begin{array}{c}\text { Kotrekhova, } 2008 \\
{[71]}\end{array}$ & $\begin{array}{c}\text { A 61-year-old male with } \\
\text { inguino-femoral skin fold mycosis } \\
\text { due to T. rubrum }\end{array}$ & Topic ISZ plus DFC for 4 weeks & $\begin{array}{l}\text { Clinical improvement and } \\
\text { eradication }\end{array}$ \\
\hline Lacaz et al., 1999 [72] & $\begin{array}{l}\text { One patient with } \\
\text { dermatophytosis caused by } \\
\text { T. raubitschekii }\end{array}$ & $\begin{array}{l}\text { FLU } 150 \text { mg per os/week for } 4 \text { weeks } \\
\text { plus topical ISZ }\end{array}$ & $\begin{array}{l}\text { Recurrence of lesions after } \\
\text { the medication was } \\
\text { discontinued. }\end{array}$ \\
\hline Lee et al., 2008 [73] & $\begin{array}{l}\text { A } 68 \text {-year-old male teacher with } \\
\text { tinea corporis due to T. rubrum }\end{array}$ & $\begin{array}{l}\text { Two treatments: topical cream } \\
\text { containing a combination of CTZ } 10 \mathrm{mg} \\
\text { and HDC for } 3 \text { weeks; topical cream } \\
\text { ISZ plus DFV for } 2 \text { weeks. }\end{array}$ & $\begin{array}{l}\text { Recurrence of skin } \\
\text { infection after the first } \\
\text { treatment; improvement } \\
\text { with cream ISZ/DFV }\end{array}$ \\
\hline Lin et al., 2014 [74] & $\begin{array}{l}\text { A 58-year-old male with } \\
\text { Trichophyton spp. Endoftalmitis }\end{array}$ & $\begin{array}{c}\text { Intravitreal AMP B } 5 \mu \mathrm{g} / 0.1 \mathrm{~mL} \\
\text { injection and oral VOR } 200 \mathrm{mg} \text { twice } \\
\text { daily + surgery }\end{array}$ & $\begin{array}{l}\text { Visual acuity } \\
\text { improvement }\end{array}$ \\
\hline $\begin{array}{l}\text { Papini et al., } 2004 \\
\text { [75] }\end{array}$ & $\begin{array}{c}\text { A 22-year-old black male student } \\
\text { with onychomycosis due to } T \text {. } \\
\text { raubitschekii }\end{array}$ & $\begin{array}{l}\text { Oral TER } 250 \mathrm{mg} / \text { day and CYC nail } \\
\text { lacquer for } 8 \text { weeks. }\end{array}$ & Full recovery \\
\hline $\begin{array}{l}\text { Pietrzak et al., } 2012 \\
\text { [76] }\end{array}$ & $\begin{array}{c}\text { A woman with dermatophytosis } \\
\text { of the thighs due to } T \text {. } \\
\text { mentagrophytes }\end{array}$ & $\begin{array}{c}\text { ISZ and DFV; cryotherapy with liquid } \\
\text { nitrogen was started after antifungal } \\
\text { therapy, for persistent lesions of } \\
\text { the skin }\end{array}$ & $\begin{array}{c}\text { Direct microscopic } \\
\text { mycologic examination } \\
\text { and culture on BioMerieux } \\
\text { medium were negative; } \\
\text { however, the lesions } \\
\text { persisted, assuming a } \\
\text { completely different } \\
\text { aspect. recovery after } \\
\text { cryotherapy. }\end{array}$ \\
\hline $\begin{array}{l}\text { Markey et al., } 2003 \\
\text { [77] }\end{array}$ & $\begin{array}{c}\text { Two young sisters, ages } 5 \text { and } 6 \\
\text { years with tinea capitis due to } T \text {. } \\
\text { soudanense }\end{array}$ & $\begin{array}{c}\text { GRI } 15 \mathrm{mg} / \mathrm{kg} / \text { day and } 2.5 \% \text { SES } \\
\text { lotion as a shampoo twice a week for } \\
8 \text { weeks for the tinea capitis }\end{array}$ & Full recovery \\
\hline $\begin{array}{l}\text { Calabrò et al., } 2011 \\
\text { [78] }\end{array}$ & $\begin{array}{c}\text { A 26-year-old man born in } \\
\text { Senegal, but living in Naples for } \\
\text { seven months with T. violaceum } \\
\text { infection }\end{array}$ & $\begin{array}{l}\text { Systemic treatment with GRI at } \\
15 \mathrm{mg} / \mathrm{kg} / \text { day and topical with TIO } 1 \% \\
\text { Twice a day for one month were } \\
\text { administered. }\end{array}$ & Full recovery \\
\hline Balci et al., 2008 [79] & $\begin{array}{l}\text { A 54-year-old immunocompetent } \\
\text { female with widespread, chronic, } \\
\text { and fluconazole-resistant } T . \\
\text { rubrum Infection }\end{array}$ & Systemic ITZ and SRZ cream & Full Recovery \\
\hline $\begin{array}{l}\text { Veraldi et al., } 2015 \\
\text { [80] }\end{array}$ & $\begin{array}{l}\text { A 47-year-old Italian woman with } \\
\text { tinea imbricata located on the } \\
\text { thighs and legs due to } \\
\text { T. concentricum }\end{array}$ & $\begin{array}{l}\text { GRI } 1 \mathrm{~g} / \text { day for } 6 \text { weeks and } 1 \% \text { TER } \\
\text { cream } 2 \text { applications/day for } 6 \text { weeks }\end{array}$ & Full recovery \\
\hline
\end{tabular}


Table 3. Cont

\begin{tabular}{|c|c|c|c|}
\hline Reference & Number of Isolates and Species & Combinations & Results \\
\hline Yin, et al., 2013 [81] & $\begin{array}{l}\text { Three familial cases with tinea } \\
\text { capitis and tinea corporis due to } \\
\text { M. canis }\end{array}$ & $\begin{array}{l}\text { Oral TER + cream containing } 1 \% \text { NAF } \\
025 \% \text { KTZ-100 mg/day ITZ + cream } \\
\text { containing } 1 \% \text { NAF } 025 \% \text { KTZ }\end{array}$ & Full recovery \\
\hline Zhan et al., 2015 [82] & $\begin{array}{l}\text { A } 48 \text {-year-old female with a } \\
\text { chronic disseminated } \\
\text { dermatophytosis due to } \\
\text { T. violaceum }\end{array}$ & $\begin{array}{l}\text { TER } 0.25 \mathrm{~g} / \text { day, } 1 \% \text { TER gel for } \\
\text { external use and } 2 \% \text { KTZ lotion for } \\
\text { shampoo and bath }\end{array}$ & $\begin{array}{l}\text { A sufficient decrease of the } \\
\text { scalp and skin damage } \\
\text { after } 4 \text { weeks, but no } \\
\text { improvement of the nails, } \\
\text { and after that, the patients } \\
\text { was lost to follow-up. }\end{array}$ \\
\hline
\end{tabular}

\begin{tabular}{cc}
\hline $\begin{array}{c}\text { Zhang et al., 2009 } \\
\text { [83] }\end{array}$ & $\begin{array}{c}\text { Three family members with } \\
\text { kerion and tinea corporis due to } \\
\text { T. mentagrophytes }\end{array}$ \\
\hline $\begin{array}{c}\text { Zhang et al., 2015 } \\
\text { [84] }\end{array}$ & $\begin{array}{c}\text { A 54-year-old Chinese male } \\
\text { patient with generalized } \\
\text { superficial mycosis caused by } \\
\text { T. raubitschekii }\end{array}$ \\
\hline $\begin{array}{c}\text { Zhuang et al., 2016 } \\
\text { [85] }\end{array}$ & $\begin{array}{c}\text { An 18-year-old girl with tinea } \\
\text { faciei on the right eyebrow caused } \\
\text { by T. mentagrophytes }\end{array}$ \\
\end{tabular}

ITZ 100 mg/day plus KTZ shampoo 2\%

$$
+3 \text { months }
$$

Clinical cure

TER 250mg/day and topical NHY and KTZ cream, containing 1\% NHY and $0.25 \% \mathrm{KTZ}$.
Full recovery

TER $250 \mathrm{mg} /$ day combined with daily topical use of $1 \%$ naftifine- $0.25 \%$

ketoconazole cream, after washing the lesion with $2 \%$ ketoconazole shampoo.

Case 2: hourly topical NAT $50 \mathrm{mg} / \mathrm{mL}$ and OFL $3 \mathrm{mg} / \mathrm{mL} 4$ times daily and oral FLU $200 \mathrm{mg}$ twice daily. Case 3: topical AMP B $10 \mathrm{mg} / \mathrm{mL}$ every $30 \mathrm{~min}$ for 1 day and hourly thereafter, MIZ

$10 \mathrm{mg} / \mathrm{mL}$ hourly, and OFL $3 \mathrm{mg} / \mathrm{mL}$ 4 times daily, along with oral FLU $200 \mathrm{mg}$ twice daily. Case 4: hourly topical MIZ $10 \mathrm{mg} / \mathrm{mL}$, oral FLU
Abdulkarim et al., $\quad$ Five cases report of fungal 2006 [86] keratitis caused by T. schoenleinii
Romano et al., 2014 [87]
18 children and 18 adults with infections due to T. violaceum
$200 \mathrm{mg}$ twice daily for 3 days and once daily thereafter. Because of a worsening clinical course, topical AMP B $5 \mathrm{mg} / \mathrm{mL}$ was added hourly. Case 5: hourly topical NAT $50 \mathrm{mg} / \mathrm{mL}$ and oral FLU $200 \mathrm{mg}$ twice daily. Following gradual improvement in the stromal infiltrate, cessation of further stromal thinning, and resolution of the hypopyon.

The 13 index cases and the 16 patients infected by them were treated with $10 \int \mathrm{mg} / \mathrm{kg}$ day GRI for 45 days and topical IMZ for 20-30 days. 23 adults with spreading tinea corporis were treated with $100 \mathrm{mg}$ ITZ for 15-20 days and those with tinea capitis with the same dose of the antimycotic for 45 days and with topical IMZ for 15-20 days, depending on the number of patches.

A total of 254 patients with tinea inguinalis, corporis, pedis, manus:

Erbakan et al., 1974 [88] 69 T. rubrum, 31 T. mentagrophytes, 7 T. violaceum, 18 E. floccousm; $6 \mathrm{M}$. canis; no growth in the remaining cases
Topical (i.e., Wilkinson's salve, iodize alcohol, undecylenic acid,

5-bromo-4'-chlorosalicylanilide, tolnaftate) plus GRI topical vs GRI alone
Improvement

Full recovery

Topical treatment plus GRI possibly enhances the healing capacity and shortens the time of treatment but no effect in the recurrences 
Table 3. Cont

\begin{tabular}{|c|c|c|c|}
\hline Reference & Number of Isolates and Species & Combinations & Results \\
\hline Baran et al., 2007 [89] & $\begin{array}{l}\text { Clinical trial AMF plus TER vs. } \\
\text { TER alone in } 249 \text { patients with } \\
\text { onychomycosis with matrix } \\
\text { involvement due to T. rubrum > } \\
90 \% \text { of cases }\end{array}$ & $\begin{array}{c}\text { AMF nail lacquer once weekly for } \\
12 \text { months plus TER } 250 \mathrm{mg} \text { once daily } \\
\text { for } 3 \text { months }\end{array}$ & $\begin{array}{l}\text { Higher success rate for } \\
\text { patients in combination } \\
\text { therapy: } 59.2 \% \text { vs } 45 \%\end{array}$ \\
\hline $\begin{array}{c}\text { Hussain et al., } 1999 \\
\text { [90] }\end{array}$ & $\begin{array}{l}\text { Clinical trial PRE plus GRI in } \\
30 \text { patients with Trichophyton } \\
\text { infection }\end{array}$ & Oral GRI and oral PRE & No difference \\
\hline Baran, 2001 [91] & $\begin{array}{c}\text { Clinical trial AMF plus ITZ vs ITZ } \\
\text { in } 131 \text { patients with T. rubrum in } \\
\text { the majority of cases }\end{array}$ & $\begin{array}{l}15 \text { months of once-weekly topical AMF } \\
\text { lacquer in combination with } 6 \text { weeks } \\
\text { (group at } 6 \text { ) or } 12 \text { weeks (group at 12) of } \\
\text { oral TER } 250 \mathrm{mg} \text { once daily }\end{array}$ & $\begin{array}{l}\text { AMF plus TER is more } \\
\text { effective than TER alone }\end{array}$ \\
\hline
\end{tabular}

ITZ, itraconazole; LUZ, luliconazole; EBE, eberconazole; TER, terbinafine; CCL, ciclopirox; MTP, methylprednisolone; NHY, naftifine hydrochloride; GRI, griseofulvin; AMP B, amphotericin B; AMR, amorolfine; VOR, voriconazole; ISN, isoconazole nitrate; DFV, diflucortolone valerate; ISZ, isoconazole; DFC, difluocortolone; CTZ, clotrimazole; HDC, hydrocortisone; NAF, naftifine; CYC, ciclopirox olamine; IMZ, imidazole; SRZ, sertaconazole nitrate; TIO, tioconazole; SES, selenium sulphide; NAT, natamycin; OFL, ofloxacin; PRE, prednisolone. M., Microsporum; T., Trichophyton; E., Epidermophyton.

\section{Conclusions}

Although dermatophyte infections are rarely life threatening, their chronicity and the frequency of relapse require prolonged treatment, resulting in an increased risk of drug toxicity and development of drug resistance. Similarly to what has been already observed in systemic fungal infections sustained by Candida spp. or Aspergillus spp., emergence of drug resistant strains among isolates of Trichophyton spp. has been lately documented.

Although dermatophytes are a group of fungi quite difficult to test in vitro (i.e., slow growth, inoculum preparation, incubation intervals etc.), standardized procedures have been introduced and validated, thereby making antifungal susceptibility testing of dermatophytes easier. This has led to experimenting with various pharmacological associations aimed at increasing the efficacy of the therapy against this group of fungi. Most of in vitro studies investigated the combination of classic antifungal agents with several, disparate, chemical compounds. The association between an antifungal drug and plant extracts, including essential oils, seems to evoke a particular interest. The reciprocal potentiation of the molecules upon combination makes these approaches particularly appealing in clinical practice. Although the intrinsic mechanisms of antifungal activity of these natural products have not been fully investigated, several cell targets are simultaneously involved, thereby making the occurrence of resistance unlikely. Clinical data indicate that an association of antifungal agents (systemic plus topic) is effective, and it might be useful in speeding up the clinical and microbiological healing of a superficial infection. It must be noted, however, that there are few controlled/randomized clinical trials and that unequivocal conclusions cannot be drawn. Another limitation is the lack of well-characterized antifungal-resistant isolates, whose treatment could especially benefit from a combination approach.

In summary, antifungal combinations against dermatophytes have gained considerable scientific interest over the years. To establish whether this approach can become a reliable treatment option, additional in vitro and clinical data are warranted.

Author Contributions: Conceptualization F.B., methodology L.B., S.F., G.M. and F.B., investigation L.B., S.F. and G.M., writing-original draft preparation L.B., S.F., G.M. and F.B., writing-review and editing F.B. All authors have read and agreed to the present version of the manuscript.

Funding: This research received no external funding.

Institutional Review Board Statement: Not applicable.

Informed Consent Statement: Not applicable. 
Acknowledgments: Not applicable.

Conflicts of Interest: The authors declare no conflict of interest.

\section{References}

1. Weitzman, I.; Summerbell, R.C. The dermatophytes. Clin. Microbiol. Rev. 1995, 8, 240-259. [CrossRef]

2. Gräser, Y.; Monod, M.; Bouchara, J.-P.; Dukik, K.; Nenoff, P.; Kargl, A.; Kupsch, C.; Zhan, P.; Packeu, A.; Chaturvedi, V.; et al. New insights in dermatophyte research. Med. Mycol. 2018, 56 (Suppl. 1), S2-S9. [CrossRef] [PubMed]

3. De Hoog, G.S.; Dukik, K.; Monod, M.; Packeu, A.; Stubbe, D.; Hendrickx, M.; Kupsch, C.; Stielow, J.B.; Freeke, J.; Göker, M.; et al. Toward a Novel Multilocus Phylogenetic Taxonomy for the Dermatophytes. Mycopathologia 2016, 182, 5-31. [CrossRef]

4. Degreef, H. Clinical Forms of Dermatophytosis (Ringworm Infection). Mycopathologia 2008, 166, 257-265. [CrossRef] [PubMed]

5. Chermette, R.; Ferreiro, L.; Guillot, J. Dermatophytoses in Animals. Mycopathologia 2008, 166, 385-405. [CrossRef]

6. Gupta, A.K.; Cooper, E.A. Update in Antifungal Therapy of Dermatophytosis. Mycopathologia 2008, 166, 353-367. [CrossRef] [PubMed]

7. Petersen, A.B.; Rønnest, M.H.; Larsen, T.O.; Clausen, M.H. The Chemistry of Griseofulvin. Chem. Rev. 2014, 114, 12088-12107. [CrossRef]

8. Nivoix, Y.; LeDoux, M.-P.; Herbrecht, R. Antifungal Therapy: New and Evolving Therapies. Semin. Respir. Crit. Care Med. 2020, 41, 158-174. [CrossRef]

9. Mueller, S.W.; Kedzior, S.K.; Miller, M.A.; Reynolds, P.M.; Kiser, T.H.; Krsak, M.; Molina, K.C. An overview of current and emerging antifungal pharmacotherapy for invasive fungal infections. Expert Opin. Pharmacother. 2021, 22, 1355-1371. [CrossRef]

10. Lopes, A.I.R.; Tavaria, F.; Pintado, M.E. Conventional and natural compounds for the treatment of dermatophytosis. Med. Mycol. 2020, 58, 707-720. [CrossRef]

11. Gupta, A.K.; Foley, K.A.; Versteeg, S.G. New Antifungal Agents and New Formulations Against Dermatophytes. Mycopathologia 2017, 182, 127-141. [CrossRef]

12. Saunte, D.M.L.; Hare, R.K.; Jørgensen, K.M.; Jørgensen, R.; Deleuran, M.; Zachariae, C.O.; Thomsen, S.F.; Bjørnskov-Halkier, L.; Kofoed, K.; Arendrup, M.C. Emerging Terbinafine Resistance in Trichophyton: Clinical Characteristics, Squalene Epoxidase Gene Mutations, and a Reliable EUCAST Method for Detection. Antimicrob. Agents Chemother. 2019, 63, 1-9. [CrossRef]

13. Arora, P.; Sardana, K.; Kaur, R.; Goyal, R.; Ghunawat, S. Is antifungal resistance a cause for treatment failure in dermatophytosis: A study focused on tinea corporis and cruris from a tertiary centre? Indian Dermatol. Online J. 2018, 9, 90-95. [CrossRef]

14. Singh, A.; Masih, A.; Khurana, A.; Singh, P.K.; Gupta, M.; Hagen, F.; Meis, J.F.; Chowdhary, A. High terbinafine resistance in Trichophyton interdigitale isolates in Delhi, India harbouring mutations in the squalene epoxidase gene. Mycoses 2018, 61, 477-484. [CrossRef]

15. Khurana, A.; Sardana, K.; Chowdhary, A. Antifungal resistance in dermatophytes: Recent trends and therapeutic implications. Fungal Genet. Biol. 2019, 132, 103255. [CrossRef]

16. Yamada, T.; Maeda, M.; Alshahni, M.M.; Tanaka, R.; Yaguchi, T.; Bontems, O.; Salamin, K.; Fratti, M.; Monod, M. Terbinafine Resistance of Trichophyton Clinical Isolates Caused by Specific Point Mutations in the Squalene Epoxidase Gene. Antimicrob. Agents Chemother. 2017, 61, e00115-17. [CrossRef]

17. Shen, J.J.; Arendrup, M.C.; Verma, S.; Saunte, D.M.L. The Emerging Terbinafine-Resistant Trichophyton Epidemic: What Is the Role of Antifungal Susceptibility Testing? Dermatology 2021, 31, 1-20. [CrossRef] [PubMed]

18. CLSI. Reference Method for Broth Dilution Antifungal Susceptibility Testing of Filamentous Fungi; Approved Standard. CLSI Document M38- ACLSI, Wayne, PA. [Internet]. April 2008. Available online: https://clsi.org/me-dia/1455/m38a2_sample.pdf (accessed on 1 June 2021).

19. Arendrup, M.C.; Kahlmeter, G.; Guinea, J.; Meletiadis, J. How to: Perform antifungal susceptibility testing of microconid-iaforming dermatophytes following the new reference EUCAST method E.Def 11.0, exemplified by Trichophyton. Clin. Microbiol. Infect. 2021, 27, 55-60. [CrossRef]

20. Arendrup, M.C.; Jørgensen, K.M.; Guinea, J.; Lagrou, K.; Chryssanthou, E.; Hayette, M.-P.; Barchiesi, F.; Lass-Flörl, C.; Hamal, P.; Dannaoui, E.; et al. Multicentre validation of a EUCAST method for the antifungal susceptibility testing of microconidia-forming dermatophytes. J. Antimicrob. Chemother. 2020, 75, 1807-1819. [CrossRef] [PubMed]

21. Su, S.; Yan, H.; Min, L.; Wang, H.; Chen, X.; Shi, J.; Sun, S. The antifungal activity of caspofungin in combination with antifungals or non-antifungals against Candida species In Vitro and in clinical therapy. Expert Rev. Anti-Infect. Ther. 2021, 28, 1-18. [CrossRef] [PubMed]

22. Gupta, A.K.; Venkataraman, M.; Renaud, H.J.; Summerbell, R.; Shear, N.H.; Piguet, V. The increasing problem of treatmentresistant fungal infections: A call for antifungal stewardship programs. Int. J. Dermatol. 2021, 17. [CrossRef]

23. Moher, D.; Liberati, A.; Tetzlaff, J.; Altman, D.G.; PRISMA Group. Preferred reporting items for systematic reviews and meta-analyses: The PRISMA statement. BMJ 2009, 339, b2535. [CrossRef]

24. Banič, S.; Lunder, M. Additive Effect of the combination of griseofulvin and ketoconazole against Microsporum canis in vitro. Mycoses 1989, 32, 487-489. [CrossRef]

25. Harman, S.; Ashbee, H.R.; Evans, E.G.V. Testing of antifungal combinations against yeasts and dermatophytes. J. Dermatol. Treat. 2004, 15, 104-107. [CrossRef] [PubMed] 
26. Laurent, A.; Monod, M. Production ofTrichophyton rubrummicrospores in large quantities and its application to evaluate amorolfine/azole compound interactions In Vitro. Mycoses 2017, 60, 581-586. [CrossRef] [PubMed]

27. Polak, A. Combination of amorolfine with various antifungal drugs in dermatophytosis. Mycoses 1993, 36, 43-49. [CrossRef] [PubMed]

28. Santos, D.A.; Hamdan, J.S. In Vitro antifungal oral drug and drug-combination activity against onychomycosis causative dermatophytes. Med. Mycol. 2006, 44, 357-362. [CrossRef] [PubMed]

29. Sugiura, K.; Masumoto, A.; Tachibana, H.; Tatsumi, Y. In Vitro Combination Effect of Topical and Oral Anti-Onychomycosis Drugs on Trichophyton rubrum and Trichophyton interdigitale. J. Fungi 2021, 7, 208. [CrossRef] [PubMed]

30. Tamura, T.; Asahara, M.; Yamamoto, M.; Yamaura, M.; Matsumura, M.; Goto, K.; Rezaei-Matehkolaei, A.; Mirhendi, H.; Makimura, M.; Makimura, K. In Vitro susceptibility of dermatomycoses agents to six antifungal drugs and evaluation by fractional inhibitory concentration index of combined effects of amorolfine and itraconazole in dermatophytes. Microbiol. Immunol. 2014, 58, 1-8. [CrossRef] [PubMed]

31. Danielli, L.J.; Pippi, B.; Duarte, J.A.; Maciel, A.J.; Lopes, W.; Machado, M.; Oliveira, L.F.S.; Vainstein, M.; Teixeira, M.L.; Bordignon, S.A.L.; et al. Antifungal mechanism of action of Schinus lentiscifolius Marchand essential oil and its synergistic effect In Vitro with terbinafine and ciclopirox against dermatophytes. J. Pharm. Pharmacol. 2018, 70, 1216-1227. [CrossRef] [PubMed]

32. Dias, N.; Dias, M.; Cavaleiro, C.; Sousa, M.D.C.R.; Lima, N.; Machado, M. Oxygenated monoterpenes-rich volatile oils as potential antifungal agents for dermatophytes. Nat. Prod. Res. 2016, 31, 460-464. [CrossRef]

33. Ala, F.; Yusuf, U.K.; Jamal, F.; Khodavandi, A. In Vitro antifungal activity of allicin alone and in combination with two med-ications against Trichophyton rubrum. World J. Microbiol. Biotechnol. 2010, 26, 2193-2198. [CrossRef]

34. Galgóczy, L.; Papp, T.; Pócsi, I.; Hegedûs, N.; Vágvölgyi, C. In Vitro activity of Penicillium chrysogenum antifungal protein (PAF) and its combination with fluconazole against different dermatophytes. Antonie Leeuwenhoek 2008, 94, 463-470. [CrossRef]

35. Houël, E.; Rodrigues, A.M.S.; Jahn-Oyac, A.; Bessière, J.-M.; Eparvier, V.; Deharo, E.; Stien, D. In Vitro antidermatophytic activity of Otacanthus azureus (Linden) Ronse essential oil alone and in combination with azoles. J. Appl. Microbiol. 2014, 116, 288-294. [CrossRef]

36. Khan, M.S.A.; Ahmad, I. Antifungal activity of essential oils and their synergy with fluconazole against drug-resistant strains of Aspergillus fumigatus and Trichophyton rubrum. Appl. Microbiol. Biotechnol. 2011, 90, 1083-1094. [CrossRef] [PubMed]

37. Khan, M.S.A.; Ahmad, I.; Cameotra, S.S. Carum copticum and Thymus vulgaris oils inhibit virulence in Trichophyton rubrum and Aspergillus spp. Braz. J. Microbiol. 2014, 45, 523-531. [CrossRef]

38. Khoury, M.; El Beyrouthy, M.; Ouaini, N.; Eparvier, V.; Stien, D. Hirtellina lobelii DC. essential oil, its constituents, its combination with antimicrobial drugs and its mode of action. Fitoterapia 2019, 133, 130-136. [CrossRef] [PubMed]

39. Maciel, A.J.; Lacerda, C.P.; Danielli, L.J.; Bordignon, S.A.L.; Fuentefria, A.M.; Apel, M.A. Antichemotactic and Antifungal Action of the Essential Oils from Cryptocarya aschersoniana, Schinus terebinthifolia, and Cinnamomum amoenum. Chem. Biodivers. 2019, 16, e1900204. [CrossRef] [PubMed]

40. Pyun, M.-S.; Shin, S. Antifungal effects of the volatile oils from Allium plants against Trichophyton species and synergism of the oils with ketoconazole. Phytomedicine 2006, 13, 394-400. [CrossRef]

41. Roana, J.; Mandras, N.; Scalas, D.; Campagna, P.; Tullio, V. Antifungal Activity of Melaleuca alternifolia Essential Oil (TTO) and Its Synergy with Itraconazole or Ketoconazole against Trichophyton rubrum. Molecules 2021, 26, 461. [CrossRef]

42. Rodriguez, M.V.; Sortino, M.A.; Ivancovich, J.J.; Pellegrino, J.M.; Favier, L.S.; Raimondi, M.P.; Gattuso, M.A.; Zacchino, S.A. Detection of synergistic combinations of Baccharis extracts with Terbinafine against Trichophyton rubrum with high throughput screening synergy assay (HTSS) followed by 3D graphs. Behavior of some of their components. Phytomedicine 2013, 20, 1230-1239. [CrossRef]

43. Shin, S.; Lim, S. Antifungal effects of herbal essential oils alone and in combination with ketoconazole against Trichophyton spp. J. Appl. Microbiol. 2004, 97, 1289-1296. [CrossRef] [PubMed]

44. Shin, S. Essential oil compounds from Agastache rugosa as antifungal agents against Trichophyton species. Arch. Pharmacal Res. 2004, 27, 295-299. [CrossRef] [PubMed]

45. Sim, Y.; Shin, S. Combinatorial anti-Trichophyton effects of Ligusticum chuanxiong essential oil components with antibiotics. Arch. Pharmacal. Res. 2008, 31, 497-502. [CrossRef]

46. Soares, L.A.; Gullo, F.P.; Sardi, J.D.C.O.; Pitangui, N.D.S.; Costa-Orlandi, C.B.; Sangalli-Leite, F.; Scorzoni, L.; Regasini, L.O.; Petrônio, M.S.; Souza, P.F.; et al. Anti-Trichophyton activity of protocatechuates and their synergism with fluconazole. Evid. Based Complement. Altern. Med. 2014, 2014, 1-9. [CrossRef] [PubMed]

47. Tiwari, N.; Pandit, R.; Gaikwad, S.; Gade, A.; Rai, M. Biosynthesis of zinc oxide nanoparticles by petals extract of Rosa indica L.; its formulation as nail paint and evaluation of antifungal activity against fungi causing onychomycosis. IET Nanobiotechnology 2017, 11, 205-211. [CrossRef]

48. Tullio, V.; Roana, J.; Scalas, D.; Mandras, N. Evaluation of the Antifungal Activity of Mentha x piperita (Lamiaceae) of Pancalieri (Turin, Italy) Essential Oil and Its Synergistic Interaction with Azoles. Molecules 2019, 24, 3148. [CrossRef] [PubMed]

49. Vörös-Horváth, B.; Das, S.; Salem, A.; Nagy, S.; Böszörményi, A.; Kőszegi, T.; Pál, S.; Széchenyi, A. Formulation of Tioconazole and Melaleuca alternifolia Essential Oil Pickering Emulsions for Onychomycosis Topical Treatment. Molecules 2020, $25,5544$. [CrossRef] 
50. Onyewu, C.; Eads, E.; Schell, W.A.; Perfect, J.R.; Ullmann, Y.; Kaufman, G.; Horwitz, B.A.; Berdicevsky, I.; Heitman, J. Targeting the Calcineurin Pathway Enhances Ergosterol Biosynthesis Inhibitors against Trichophyton mentagrophytes In Vitro and in a Human Skin Infection Model. Antimicrob. Agents Chemother. 2007, 51, 3743-3746. [CrossRef]

51. Ozawa, H.; Okabayashi, K.; Kano, R.; Watanabe, S.; Hasegawa, A. Antifungal Activities of the Combination of Tacrolimus and Itraconazole Against Trichophyton mentagrophytes. J. Vet. Med. Sci. 2005, 67, 629-630. [CrossRef]

52. Zhang, J.; Tan, J.; Yang, L.; He, Y. Tacrolimus, not triamcinolone acetonide, interacts synergistically with itraconazole, terbinafine, bifonazole, and amorolfine against clinical dermatophyte isolates. J. Mycol. Med. 2018, 28, 612-616. [CrossRef]

53. Simonetti, O.; Arzeni, D.; Ganzetti, G.; Silvestri, C.; Cirioni, O.; Gabrielli, E.; Castelletti, S.; Kamysz, W.; Kamysz, E.; Scalise, G.; et al. In Vitro activity of the lipopeptide derivative (Pal-lys-lys-NH2), alone and in combination with antifungal agents, against clinical isolates of dermatophytes. Br. J. Dermatol. 2009, 161, 249-252. [CrossRef] [PubMed]

54. Simonetti, O.; Ganzetti, G.; Arzeni, D.; Campanati, A.; Marconi, B.; Silvestri, C.; Cirioni, O.; Gabrielli, E.; Lenci, I.; Kamysz, W.; et al. In Vitro activity of Tachyplesin III alone and in combination with terbinafine against clinical isolates of dermatophytes. Peptides 2009, 30, 1794-1797. [CrossRef]

55. Simonetti, O.; Silvestri, C.; Arzeni, D.; Cirioni, O.; Kamysz, W.; Conte, I.; Staffolani, S.; Orsetti, E.; Morciano, A.; Castelli, P.; et al. In Vitro activity of the protegrin IB-367 alone and in combination compared with conventional antifungal agents against dermatophytes. Mycoses 2013, 57, 233-239. [CrossRef]

56. Moriello, K.A.; Verbrugge, M. Use of isolated infected spores to determine the sporocidal efficacy of two commercial antifungal rinses against Microsporum canis. Veter. Dermatol. 2007, 18, 55-58. [CrossRef] [PubMed]

57. Perrins, N.; Bond, R. Synergistic inhibition of the growth in vitro of Microsporum canis by miconazole and chlorhexidine. Vet. Dermatol. 2003, 14, 99-102. [CrossRef] [PubMed]

58. Perrins, N.; Howell, S.A.; Moore, M.; Bond, R. Inhibition of the growth In Vitro of Trichophyton mentagrophytes, Trichophyton erinacei and Microsporum persicolor by miconazole and chlorhexidine. Veter. Dermatol. 2005, 16, 330-333. [CrossRef]

59. Nyilasi, I.; Kocsubé, S.; Krizsán, K.; Galgóczy, L.; Papp, T.; Pesti, M.; Nagy, K.; Vágvölgyi, C. Susceptibility of clinically important dermatophytes against statins and different statin-antifungal combinations. Med. Mycol. 2014, 52, 1-9. [CrossRef]

60. Aneke, C.I.; Rhimi, W.; Otranto, D.; Cafarchia, C. Synergistic Effects of Efflux Pump Modulators on the Azole Antifungal Susceptibility of Microsporum canis. Mycopathologia 2020, 185, 1-10. [CrossRef]

61. Adamski, Z.; Kowalczyk, M.J.; Adamska, K.; Kubisiak-Rzepczyk, H.; Bowszyc-Dmochowska, M.; Banaszak, A.; Bartkiewicz, P.; Żaba, R. The First Non-African Case of Trichophyton rubrum var. raubitschekii or a Urease-Positive Trichophyton rubrum in Central Europe? Mycopathologia 2014, 178, 91-96. [CrossRef]

62. Budihardja, D.; Freund, V.; Mayser, P. Widespread erosive tinea corporis by Arthroderma benhamiae in a renal transplant recipient: Case report. Mycoses 2010, 53, 530-532. [CrossRef] [PubMed]

63. Czaika, V.A. Misdiagnosed zoophile tinea faciei and tinea corporis effectively treated with isoconazole nitrate and diflucortolone valerate combination therapy. Mycoses 2013, 56 (Suppl. 1), 26-29. [CrossRef]

64. Durant, J.-F.; Fonteyne, P.-A.; Richez, P.; Marot, L.; Belkhir, L.; Tennstedt, D.; Gala, J.-L. Real-time PCR and DNA sequencing for detection and identification of Trichophyton rubrumas a cause of culture negative chronic granulomatous dermatophytosis. Med. Mycol. 2009, 47, 508-514. [CrossRef]

65. Fabrizi, V.; Zacconi, I.; Principato, M.; Pesca, C.; Cruciani, D.; Crotti, S.; Papini, M. Toenail onychomycosis by Trichophyton rubrum and concurrent infestation with Tyrophagus putrescentiae. Infez. Med. 2017, 25, 377-380. [PubMed]

66. Ghislanzoni, M. Tinea incognito due to Trichophyton rubrum responsive to topical therapy with isoconazole plus corticosteroid cream. Mycoses 2008, 51 (Suppl. 4), 39-41. [CrossRef]

67. Hsieh, A.; Quenan, S.; Riat, A.; Toutous-Trellu, L.; Fontao, L. A new mutation in the SQLE gene of Trichophyton mentagrophytes associated to terbinafine resistance in a couple with disseminated tinea corporis. J. Mycol. Med. 2019, 29, 352-355. [CrossRef]

68. Jang, M.S.; Bin Park, J.; Jang, J.Y.; Yang, M.H.; Kim, J.H.; Lee, K.H.; Hwangbo, H.; Suh, K.S. Kerion celsi caused by Trichophyton erinacei from a hedgehog treated with terbinafine. J. Dermatol. 2016, 44, 1070-1071. [CrossRef] [PubMed]

69. Khaled, A.; Mbarek, L.B.; Kharfi, M.; Zeglaoui, F.; Bouratbine, A.; Fazaa, B.; Barek, M.R.K. Tinea capitis favosa due to Tri-chophyton schoenleinii. Acta Dermatovenerol. Alp. Panon. Adriat. 2007, 16, 34-36.

70. Kimura, U.; Hiruma, M.; Kano, R.; Matsumoto, T.; Noguchi, H.; Takamori, K.; Suga, Y. Caution and warning: Arrival of terbinafine-resistant Trichophyton interdigitale of the Indian genotype, isolated from extensive dermatophytosis, in Japan. J. Dermatol. 2020, 47, e192-e193. [CrossRef] [PubMed]

71. Kotrekhova, L. The effective use of isoconazole nitrate and diflucortolone valerate cream in the treatment of inguino-femoral skin fold mycosis. Mycoses 2008, 51 (Suppl. 4), 29-31. [CrossRef]

72. Lacaz, C.D.S.; Zaitz, C.; Ruiz, L.R.B.; De Souza, V.M.; Santos, A.R.A.; Muramatu, L.H.; De Melo, N.T.; Heins-Vaccari, E.M.; Hernández-Arriagada, G.L.; De Freitas-Leite, R.S. Dermatophytosis caused by Trichophyton raubitschekii. Report of the first case in São Paulo, Brazil. Rev. Inst. Med. Trop. São Paulo 1999, 41, 313-317. [CrossRef]

73. Lee, G.-Y.; Kim, W.-S. Tinea corporis of the shin and chest successfully treated with a topical antifungal and corticosteroid cream. Mycoses 2008, 51 (Suppl. 4), 34-36. [CrossRef]

74. Lin, C.-M.; Pao, S.-I.; Chen, Y.-H.; Chen, J.-T.; Lu, D.-W.; Chen, C.-L. Fungal endophthalmitis caused by Trichophyton spp. after cataract surgery. Clin. Exp. Ophthalmol. 2014, 42, 696-697. [CrossRef] [PubMed] 
75. Papini, M.; Greco, C.; Pileri, F. Onychomycosis caused by an isolate conforming to the description of Trichophyton raubitschekii. Med. Mycol. 2004, 42, 273-276. [CrossRef] [PubMed]

76. Pietrzak, A.; Tomasiewicz, K.; Kanitakis, J.; Paszkowski, T.; Dybiec, E.; Donica, H.; Wójtowicz, A.; Terlecki, P.; Chodorowska, G. Trichophyton mentagrophytes-associated Majocchi's granuloma treated with cryotherapy. Folia Histochem. Cytobiol. 2012, 50, 486-489. [CrossRef] [PubMed]

77. Markey, R.J.; Staat, M.A.; Gerrety, M.J.T.; Lucky, A.W. Tinea capitis due to Trichophyton soudanense in Cincinnati, Ohio, in internationally adopted children from Liberia. Pediatr. Dermatol. 2003, 20, 408-410. [CrossRef] [PubMed]

78. Calabrò, G.; Nino, M.; La Bella, S.; Gallo, L. Trichophyton violaceum infection in an adult black patient in Europe. Int. J. Dermatol. 2011, 50, 761-763. [CrossRef]

79. Balci, D.D.; Cetin, M. Widespread, chronic, and fluconazole-resistant Trichophyton rubrum infection in an immunocompetent patient. Mycoses 2008, 51, 546-548. [CrossRef]

80. Veraldi, S.; Pontini, P.; Nazzaro, G. A Case of Tinea Imbricata in an Italian Woman. Acta Derm. Venereol. 2015, 95, 235-237. [CrossRef]

81. Yin, B.; Xiao, Y.; Ran, Y.; Kang, D.; Dai, Y.; Lama, J. Microsporum canis Infection in Three Familial Cases with Tinea Capitis and Tinea Corporis. Mycopathologia 2013, 176, 259-265. [CrossRef]

82. Zhan, P.; Li, Z.; Geng, C.; Jiang, Q.; Jin, Y.; Dolatabadi, S.; Liu, W.; De Hoog, G.S. A Chronic Disseminated Dermatophytosis Due to Trichophyton violaceum. Mycopathologia 2014, 179, 159-161. [CrossRef]

83. Zhang, H.; Ran, Y.; Liu, Y.; Zhang, R.; Lin, X.; Yan, W.; Dai, Y. Arthroderma vanbreuseghemiiinfection in three family members with kerion and tinea corporis. Med. Mycol. 2009, 47, 539-544. [CrossRef] [PubMed]

84. Zhang, H.; Xiong, X.; Liu, T.; Ran, Y. Generalized Superficial Mycosis Caused by Trichophyton raubitschekii in China: Case Report and Review of the Literature. Mycopathologia 2015, 179, 279-284. [CrossRef]

85. Zhuang, K.W.; Dai, Y.L.; Ran, Y.P.; Lama, J.; Fan, Y.M. Tinea faciei on the right eyebrow caused by Trichophyton interdigitale. An. Bras. Dermatol. 2016, 91, 829-831. [CrossRef]

86. Mohammad, A.; Al-Rajhi, A.; Wagoner, M.D. Trichophyton Fungal Keratitis. Cornea 2006, 25, 118-122. [CrossRef]

87. Romano, C.; Feci, L.; Fimiani, M. Thirty-six cases of epidemic infections due toTrichophyton violaceumin Siena, Italy. Mycoses 2014, 57, 307-311. [CrossRef]

88. Erbakan, N.; Or, A.N.; Palali, Z.; Basaran, E. Studies on the treatment of dermatophytic infections of glabrous skin by topical treatment alone or with combination of griseofulvin for comparison. Mycopathologia 1974, 52, 291-298. [CrossRef] [PubMed]

89. Baran, R.; Sigurgeirsson, B.; De Berker, D.; Kaufmann, R.; Lecha, M.; Faergemann, J.; Kerrouche, N.; Sidou, F. A multicentre, randomized, controlled study of the efficacy, safety and cost-effectiveness of a combination therapy with amorolfine nail lacquer and oral terbinafine compared with oral terbinafine alone for the treatment of onychomycosis with matrix involvement. $\mathrm{Br}$. $\mathrm{J}$. Dermatol. 2007, 157, 149-157. [CrossRef]

90. Hussain, I.; Muzaffar, F.; Rashid, T.; Ahmad, T.J.; Jahangir, M.; Haroon, T.S. A randomized, comparative trial of treatment of kerion celsi with griseofulvin plus oral prednisolone vs. griseofulvin alone. Med. Mycol. 1999, 37, 97-99. [CrossRef] [PubMed]

91. Baran, R. Topical amorolfine for 15 months combined with 12 weeks of oral terbinafine, a cost-effective treatment for onychomycosis. Br. J. Dermatol. 2001, 145 (Suppl. 60), 15-19. [CrossRef] 